\title{
Calculations for Martensitic Phase Transformations by Quantum Mechanics and Thermodynamics
}

\author{
K. Schwarz, P. Mohn, V.L. Sliwko and P. Blaha \\ Technische Universität Wien, Institut für Technische Elektrochemie, A-1060 Vienna, Getreidemarkt 9/158, \\ Austria
}

\begin{abstract}
The martensitic phase transition (MPT) from $f c c$ to $b c c$ is studied by a new combination of established methods. A continuous change between the two phases is possible according to the Bain transition in which the c/a ratio in a body centered tetragonal structure is varied between $\sqrt{2}$ and 1 . As an example the electronic structure of $\mathrm{Sr}$ is determined by quantum mechanical calculations employing the full-potential linearized augmented plane wave (LAPW) method (as embodied in the WIEN code) in which exchange and correlation effects are described within the local spin density approximation. From these calculations we obtain the total energy as a function of the Bain variable c/a and the volume. The resulting energy surfaces $E(c / a, V)$ valid at $\mathrm{T}=0 \mathrm{~K}$ define the ground state and show e.g. the relative stability along the Bain transition. In a second step, this $\mathrm{T}=\mathrm{OK}$ quantum mechanical total energy surface is fitted to a GinzburgLandau model to study finite temperature effects by treating fluctuations of the two order parameters $\mathrm{c} / \mathrm{a}$ and $\mathrm{V}$. This combined approach allows to describe the temperature induced first order phase transition between the fcc and the bcc phase as being driven by strain fluctuations rather than by phonon softening.
\end{abstract}

\section{INTRODUCTION}

Martensitic phase transitions (MPT) have interested scientists for more than a century leading to an enormous literature, but still many open questions remain. The high complexity of temperature induced structural phase transitions is due to the combination of quantum mechanical (e.g. chemical bonding) and thermodynamic effects (finite temperature phase stability) which also appear on different length scales starting from the microscopic movement of single atoms over a mesoscopic scale on which crystal structure effects (tweed or twinning) take place up to a macroscopic (engineering) scale where industrial applications are performed (e.g. the shape memory alloys). In contrast to diffusive phase transitions the MPT is a collective phenomenon where a crystal undergoes a first order transition from an austenitic (high temperature) modification to a martensitic (low temperature) structure with a hysteretic behaviour typical for this phase transition. An overview about the multitude of physical and chemical effects which add up to what is known as a MPT is given for example in the book by Olson and Owen. ${ }^{(1)}$

The first ideas about a possible mechanism for MPTs go back to the work by Zener ${ }^{(2)}$ who suggested that a softening of the shear mode is responsible for the instability of the parent lattice. Friedel ${ }^{(3)}$ 
attempted to provide a thermodynamic argument for the MPT and proposed that these transitions are driven by the entropy which, for the high temperature phase, should increase with temperature due to a lowering of phonon frequencies. Based on these ideas the first microscopic model was given by $\operatorname{Cochran}^{(4)}$ and Anderson ${ }^{(5)}$ who formulated the soft-mode model according to which the effective frequency of one phonon branch becomes zero at a temperature $T_{M}$ triggering the phase transition. In contrast to these ideas are the models with little phonon softening which rely on anharmonicities of the phonon branches. ${ }^{(6),(7)}$ Krumhansl and Gooding ${ }^{(8)}$ proposed such a model in which anharmonicities in the order parameter of a Landau-type model are sufficient to describe a first order phase transition. The formal equivalence between magnetic and martensitic phase transitions was noted by Lindgard and Mouritsen ${ }^{(9)}$ who treated the MPT on the basis of an Ising model. An additional aspect is the kinetics associated in such a transformation. From this brief overview it becomes clear that a theoretical model which covers all properties mentioned above will be difficult to develop and is indeed not yet at hand. However, enormous progress in the understanding of this highly complex phenomenon has been made during the last years. The present paper tries to elucidate the microscopic part of the problem and is organized in two major parts: i) we investigate by means of highly precise band structure calculations the energetics of a special transition between the $\mathrm{fcc}$ and the bcc lattice in $\mathrm{Sr}$, ii) we use these quantum mechanical $\mathrm{T}=0 \mathrm{~K}$ results in a Landau-Ginzburg model with which we study the influence of fluctuations of the "geometrical" order parameters which are found to drive a first order phase transition.

If a crystal undergoes a transition from one structure into another, individual atoms in the unit cell or complete atomic planes in the whole crystal must move to new positions in an orderly fashion. Out of the 24 possible geometries for such a transition between the body centered cubic (bcc) and the face centered cubic (fcc) crystal structure the Bain transformation is by far the simplest. It is known since 1924 when E.C. Bain ${ }^{(10)}$ described this transition between the bcc and the fcc structure via a tetragonal distortion shown in Fig. 1. Both the fcc and the bcc lattice can be thought of as a special case of a body centered tetragonal (bct) unit cell.

In the fcc case $a$ and $b$ are equal and the lattice vector $c$ has the length $a \sqrt{ } 2$ while in the bcc case all three lattice vectors have equal length. The Baintransformation between the fcc and the boc lattice is thus described by the continuous change of a single variable, namely the $c / a$ ratio, so that $c / a=\sqrt{2}$ refers to the $f c c$

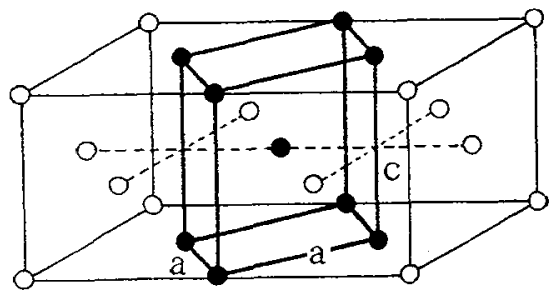

Figure 1: Body centered tetragonal (bct) cell of an fcc latlice illustrating the continous transition to the bcc lattice by varying $\mathrm{c} / \mathrm{a}$ according to the Bain path. and $c / a=1$ to the bcc structure. It should be noted, that this Bain transformation is just one possible path between these two structures and that other, more complicated transformations have also been found experimentally.

\section{COMPUTATIONAL DETAILS}

We use quantum mechanical calculations to obtain the total energy at $T=0$ on a first principles basis. We compute the electronic band structure within the density functional theory and the local density approximation (LDA) by means of the Full-Potential Linearized-Augmented-Plane-Wave (FP-LAPW) method employing the WIEN93 code published by Blaha et al. ${ }^{(1)}$ Since the structural energies are often only fractions of $1 \mathrm{mRy}$ the calculations were carried out for a sufficiently large number of $\mathbf{k}$-points in the irreducible wedge of the bct Brillouin zone (between 540 and $1800 \mathrm{k}$-points, depending on the system). A very high precision in the total energy results is necessary and was obtained by using a plane-wave cutoff $\mathrm{R} \times \mathrm{K}=9$ leading to about 140 basis functions. The total energy (at $\mathrm{T}=0$ ) is computed 
as a function of volume $\mathrm{V}$ and $\mathrm{c} / \mathrm{a}$ ratio (Bain variable), the two most important parameters.

In a previous study ${ }^{(12)}$ we have performed such LAPW calculations for selected simple metals ( $\mathbf{L i}$, $\mathrm{K}, \mathrm{Rb}, \mathrm{Ca}$, and $\mathrm{Sr}$ ) and transition metals ( $\mathrm{Ti}$ and $\mathrm{V}$ ) and discussed their similarities and differences. For each system we calculated the total energy for at least 8 different volumes and 10 different c/a ratios. From this series of self-consistent FP-LAPW calculations we derive total energy surfaces E(c/a,V). This information was used to discuss e.g. the pressure induced phase transition in $\mathrm{Ca}^{(12)}$ At this point we like to mention that this part of our work is similar to previous investigations by Chen et al. ${ }^{(13)}$ for the hcp $\leftrightarrow$ bcc transition in barium and the reader should be aware that other quantum mechanical models have been applied to this problem by various authors. ${ }^{(14)(15)}$

By fitting the FP-LAPW total energies to a polynomial, we obtain an analytic expression for the energy surface $\mathrm{E}(\mathrm{c} / \mathrm{a}, \mathrm{V})$ given by:

$$
E(c / a, V)=\sum_{i=0, n} \sum_{j=0, m} A_{i j}(c / a)^{i} V^{j}
$$

To monitor the convergence of the fit we performed a least square procedure using orthogonal Chebychev polynomials from which the final polynomial given in $\mathrm{Eq} .1$ was derived. This analytic expression allows to calculate several characteristic quantities such as the bulk modulus $B=\left(C_{11}+2 C_{12}\right) / 3$ and the tetragonal shear constant $C^{\prime}=\left(C_{11}-C_{12}\right) / 2$ which - in the cubic case - are related to the respective elastic constants $C_{11}$ and $\mathrm{C}_{12}$. The expansion given in $\mathrm{Eq} .1$ is equivalent to the soft-mode model proposed by $\mathrm{Clapp}^{(6)}$ who explaines the occurance of MPTs from nucleation due to local strains.

\section{A MEAN FIELD MODEL FOR MPTS}

It has been noticed already, that there is a formal similarity between structural and magnetic phase transitions. Lindgård and Mouritsen ${ }^{(9)}$ discussed structural phase transitions by mapping the relevant properties to an Ising model where they assume that the two structural modifications are equivalent to the a ferro- and an antiferromagnetic solution of the Ising hamiltonian. In a later paper ${ }^{(16)}$ both authors present a fluctuation model based on their magnetic hamiltonian. The anharmonic model proposed by Krumhansl and Gooding ${ }^{(8)}$ describes the MPT in terms of a Landau expansion of the free energy by assuming local displacements which are scaled to low lying phonon modes. Recently a mean field model has been presented $^{(17)}$ which treats the thermal properties on the basis of fluctuations of the mean square displacement. All these models have one thing in common, namely that they no longer rely on the softening of a certain phonon mode. Random excitations of small scale displacements are sufficient to trigger the phase transition.

To introduce the effects of finite temperature we generalize the Landau expansion given by Eq. 1 by introducing local fluctuations of the order parameters which represent the small displacements. ${ }^{(18)}$ The fluctuation of the Bain variable is denoted by $\xi(r)$ (along c/a) and the fluctuation of the volume by $v(r)$. Formally this means that we replace the order parameter c/a by $<c / a+\xi(r)>$, where $<$ denotes the statistical average over the phase space of the fluctuations in the Gaussian approximation. At $\mathrm{T}=0 \mathrm{~K}$, when the fluctuations vanish the original Landau expansion (Eq. 1) is regained. The thermal dependence of the fluctuation is calculated by determining the free energy of the fluctuation hamiltonian which is derived in analogy to the classical spin-fluctuation hamiltonian. ${ }^{(19)}$ With our ansatz we have assumed that our fluctuations can be represented by independent particles moving in a mean field. With fluctuations the energy becomes a function of four variables, $E\left(c / a, V,\left\langle\xi(r)^{2}\right\rangle,\left\langle V(r)^{2}\right\rangle\right)$ where the fluctuations are assumed to be non-centered random variables varying on a spatial scale larger than the range of interactions. In the Landau-Ginzburg expansion of the free energy, non-local effects are taken into account by the lowest 
order gradient terms and the hamiltonian reads:

$$
H=\frac{1}{V} \int d^{3} r\left\{E((c / a)+\xi(r), V+v(r))+\frac{K}{2}(\nabla \xi(r))^{2}+\frac{Q}{2}(\nabla v(r))^{2}\right\}
$$

Using this Hamiltonian we calculate the free energy via a classical partition function integral over the phase space of the fluctuations. Since this function integral cannot be calculated in general, the best possible approximation to the exact free energy is obtained from the Peierls-Feynman inequality, a procedure which essentially describes a variational derivation of a mean-field model. For the classical strain- and volume-fluctuations we obtain:

$$
\left\langle\xi^{2}(r)\right\rangle=\frac{k_{B} T V k_{c}}{2 \pi^{2} K} f\left[\sqrt{\frac{K k_{c}^{2}}{2 \frac{\partial \phi}{\partial\left(\xi^{2}(r)\right\rangle}}}\right]
$$

$$
\left\langle v^{2}(r)\right\rangle=\frac{k_{B} T V q_{c}}{2 \pi^{2} Q} f\left[\sqrt{\frac{Q q_{c}^{2}}{2 \frac{\partial \phi}{\left.\partial v^{2}(r)\right\rangle}}}\right]
$$

where the function $f(x)$ is given by: $f(x)=1-(\arctan (x)) / x$. The energy contribution of the fluctuations is given by the function $\phi$ defined by:

$$
\phi=\frac{1}{V} \int d^{3} r[E((c / a)+\xi(r), V+v(r))-E((c / a), V)]
$$

The equations of state are derived by requiring minimization of $\mathrm{E}$ and $\phi$ with respect to the variables c/a and V. For a given temperature, the equations must be solved self-consistently to obtain $c / a, V,\left\langle\xi^{2}(r)\right\rangle$, and $\left\langle V^{2}(r)\right\rangle$. The energy function $E(c / a, V)$ is defined by Eq.1 and is thus given by the $T=0 \mathrm{~K}$ ground state total energy calculated from the electronic band structure. However four new parameters appear in Eqs. 3,4 , namely the two "stiffness" constants $\mathrm{K}$ and $\mathrm{Q}$ and the two cut-off wave vectors $\mathrm{k}_{\mathrm{c}}$ and $\mathrm{q}_{\mathrm{c}}$. Within our classical model, the correlation function has an Ornstein-Zernicke form and the fluctuation is given by the k-space integral over the k-dependent susceptibility (we use the term susceptibility in analogy to magnetic systems also for the structural properties, but in its more general meaning as a response function), which for a system isotropic in $\mathrm{k}$ gives:

$$
\left\langle\xi^{2}(r)\right\rangle=\frac{V}{(2 \pi)^{3}} \frac{k_{B} T}{2} \int_{0}^{k_{c}} \frac{4 \pi k^{2}}{\frac{K}{2} k^{2}+\frac{\partial \phi}{\partial\left\langle\xi^{2}(r)\right\rangle}} d k
$$

and analogously for the volume fluctuation. In Eq. 6 the constant $K$ determines the width of the momentum distribution and $k_{c}$ the largest $k$-vector (shortest wavelength) of the fluctuation. The introduction of this cut-off also corrects for the shortcoming of all mean-field models to treat long and short range order interaction on the same footing. By assuming that all fluctuations of the order parameter behave in an average manner, the mean-field model neglects fluctuations of the order parameter in which nearby parts of the system, while remaining correlated with each other, deviate from the average behaviour. This is also why mean-field models usually overestimate the critical temperature. With the inclusion of the Ginzburg term in our hamiltonian (Eq.2) we have already improved for this problem but the decay of the correlation function with increasing $k$ is still too slow, so that $k_{\mathfrak{c}}$ remains as an 
independent scaling parameter for the transition temperature.

The total energy $U=F+T S$ contains contributions of both order parameters $c / a$ and $V$. The two sums in Eq. 7 are typical for our classical model and just count the number of degrees of freedom (number of waves) and cause a constant energy shift.

$$
U=E+\phi-<\xi^{2}(r)>\frac{\partial \phi}{\partial<\xi^{2}(r)>}+\frac{k_{B} T}{2} \sum_{k \leq k_{c}}-<v^{2}(r)>\frac{\partial \phi}{\partial<v^{2}(r)>}+\frac{k_{B} T}{2} \sum_{k \leq q_{c}}
$$

By ploting $U$ as a function of $\mathrm{c} / \mathrm{a}$ and $\mathrm{V}$ (for $\mathrm{T}=\mathrm{const}$.) we can study the temperature evolution of the order parameter describing the structural transition. We conjecture that the entropy contribution to the total energy stabilizes the high temperature phase so that the fluctuation model describes an entropy driven phase transition, a result that was also derived by Kerr and Rave. ${ }^{(17)}$

\section{RESULTS AND DISCUSSION}

The combination of our quantum mechanical $(\mathrm{T}=0 \mathrm{~K})$ calculations with the finite temperature Ginzburg-Landau model allows to study temperarure induced phase transitions. In this paper we apply this approach to $\mathrm{Sr}$ and show in Fig. 2 the total energy surface $\mathrm{U}(\mathrm{c} / \mathrm{a}, \mathrm{V}, \mathrm{T})$ for $\mathrm{T}=0 \mathrm{~K}$ and a temperature above the transition temperature $T_{M}$. The $T=0 \mathrm{~K}$ total energy surface (lower panel of Fig. 2) shows that two minima exist along the Bain path. Both minima appear at values of $\mathrm{c} / \mathrm{a}$ which correspond to cubic structures (see sect.1) and have almost the same total energy. For a detailed discussion of the energetics of the Bain transformation and a comparison of the results for other alkaline, earth-alkaline, or transition metals we refer to Sliwko et al. ${ }^{(12)}$ With rising temperature the c/a fluctuations flatten out the local minimum at the fecstructure $(c / a=\sqrt{2})$ which finally disappears at a temperature $T_{M}$ where a first order phase transition occurs. The order parameter $\mathrm{c} / \mathrm{a}$ changes discontinously from $\sqrt{2}$ to 1 which means that for $\mathrm{T} \geq \mathrm{T}_{\mathrm{M}}$ the bcc structure becomes stable. The upper panel of Fig. 2 shows the total energy surface at a temperature slightly above $T_{M}$, where only a single minimum at $c / a=1$ remains but an anisotropy reminiscent of the low temperature focminimum can still be seen. In our model the transition is connected to a vanishing value for C', which is the relevant elastic constant for the Bain transformation. If a

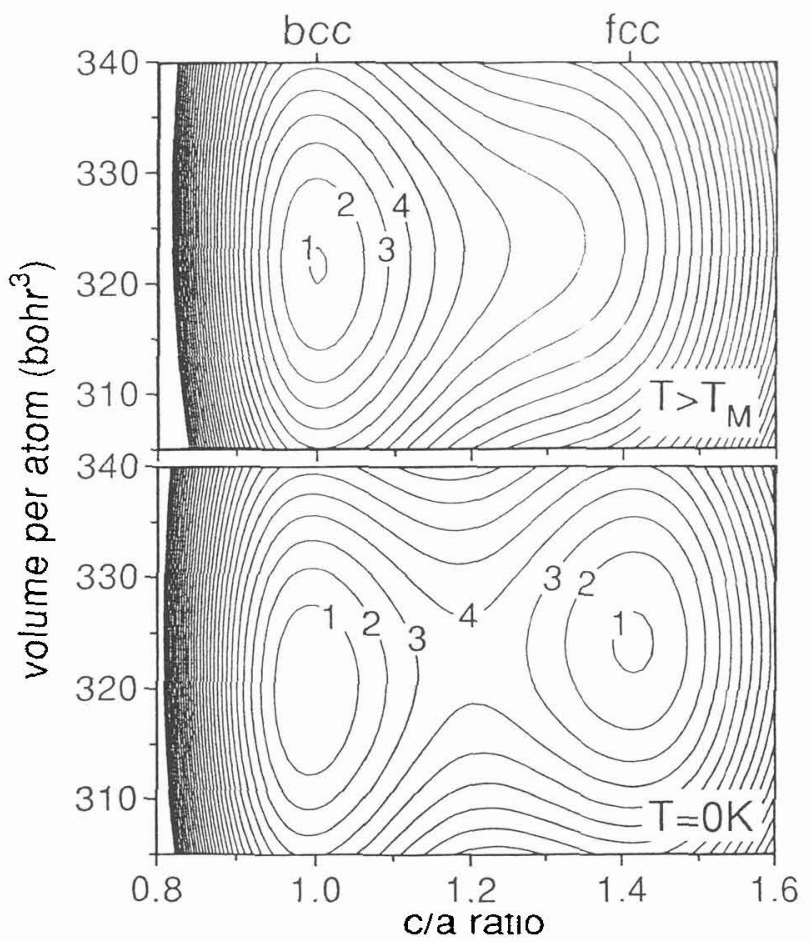

Figure 2: Energy surfaces for the Bain-path in $S r$ for $T=0 \mathrm{~K}$ (lower panel) and a temperature just above the MPT temperature $T_{M}$ (upper panel). The labels in the contour lines are in multiples of $0.1 \mathrm{mRy}$. 
different fluctuation drives the phase transition the corresponding elastic constant, or a linear combination of them must vanish.

In this paper we discuss a physical mechanism in which the atomic motions are dominated by random fluctuations whose mean square amplitudes at $\mathrm{T}_{M}$ are about $4 \%$ of the order parameter. These excitations are localized in real space but delocalized in reciprocal space while phonons show just the opposite dependence. Since for large excitation energies random fluctuations will be less favourable than collective motions (phonons), it depends on the specific energetics of a system, whether phonons or fluctuations drive a phase transition. Nowadays it is possible to compute such energies from first principles on a quantum mechanical basis. As long as the relevant order parameters can be found and we are in a regime, where fluctuations dominate, our combined model provides a possible description for a martensitic phase transition.

\section{REFERENCES}

[1] Olson G.B. and Owen W.S. eds., Martensite (ASM International, 1992).

[2] Zener C., Phys. Rev. B 71 (1947) 846.

[3] Friedel J., J. Phys. France Lent. 35 (1974) L59.

[4] Cochran W., Adv. Phys. 9 (1960) 387.

[5] Anderson P.W., Fizika Dielectrikov, edited by G.I. Skanavi, (Acad. Nauk SSR, Moscow, 1960).

[6] Clapp P.C., phys. stat. sol. (b) 57 (1973) 561.

[7] Krumhansl J.A., Solid State Commun. 48 (1992) 251.

[8] Krumhansl J.A. and Gooding R.J., Phys. Rev. B 39 (1989) 3047.

[9] Lindgård P.A. and Mouritsen O.G., Phys. Rev. Lett. 57 (1986) 2458.

[10] Bain E.C., Trans. AlME 70 (1924) 25.

[11] Blaha P., Schwarz K., and Augustyn R., Computer Code WIEN93, Technical University of Vienna, 1993 lan improved and updated Unix version of the original copyrighted WIEN code which was published by Blaha P., Schwarz K., Sorantin P. and Trickey S.B., Computer Phys. Commun. 59 (1990) 399).

[12] Sliwko V.L., Mohn P., Schwarz K. and Blaha P., Phys. Rev. B (1994) in print.

[13] Chen Y., Ho K.M. and Harmon B.N.. Phys. Rev. B 37 (1988) 283.

[14] Krasko G.L. and Olson G.B., Phys. Rev, B 40 (1989) 11536.

[15] Wentzcovich R.M. and Krakauer H., Phys. Rev. B 42 (1990) 4563.

[16] Lindgård P.A. and Mouritsen O.G., Phys. Rev. B 41 (1990) 688.

[17] Kerr W.C. and Rave M.J., Phys. Rev. B 48 (1993) 16234.

[18] Mohn P., Schwarz K., Sliwko V.L., Blaha P., Phys. Rev. B, to be published.

[19] Murata K.K. and Doniach S., Phys. Rev. Lett. 29 (1972) 285. 